\title{
Intracranial arterial stenosis associated with Hashimoto's disease: angiographic features and clinical outcomes
}

\author{
Eika Hamano, Masaki Nishimura, Hisae Mori, Tetsu Satow and Jun C. Takahashi*
}

\begin{abstract}
Background: Hashimoto's disease has reportedly been associated with stroke; however, cerebrovascular morphology and clinical course remain poorly documented. The present study aimed to determine the angiographic features and clinical outcomes of intracranial arterial stenosis (IAS) associated with Hashimoto's disease in a retrospective cohort.

Methods: Overall, 107 adult patients with IAS were screened for anti-thyroid antibodies; of these, 26 patients tested positive. The 42 affected hemispheres were classified into subgroups according to the steno-occlusion site and the development of abnormal collateral (moyamoya) vessels. These subgroups were dichotomized into moyamoya vessels positive (MM type) and negative (non-MM type). The initial presentation, IAS progression, and vascular events during the follow-up period were compared.

Results: The following sites of stenosis were identified: the bifurcation of the internal carotid artery in 11 (26.2\%), M1 or A1 in 29 (69.0\%), and more distal (M2-M4/A2-A4) in 2 (4.8\%) hemispheres. Further, 17 hemispheres were categorized into the MM type and 25 were classified into the non-MM type. During the follow-up period (mean 2.5 years), IAS progression was identified in $8(32 \%)$ hemispheres of the non-MM type and $0(0 \%)$ hemispheres of the MM type $(p=$ 0.041). Ischemic attacks occurred in 5 (20.0\%) hemispheres of the non-MM type (4.6\%/year) and 0 hemispheres of the MM type ( $p=0.08)$. Further, $4(23.5 \%)$ hemispheres of the MM type experienced intracerebral hemorrhage, whereas none of the non-MM type hemorrhaged ( $p=0.012$ ).

Conclusions: Hashimoto's disease-associated IAS exhibits various angiographic morphologies, resulting in different clinical presentations. Screening for anti-thyroid antibodies and careful management based on vascular morphology appears important in adults with IAS.
\end{abstract}

Keywords: Anti-thyroid antibody, Hashimoto's disease, Intracranial arterial stenosis, Moyamoya disease

\section{Background}

Hashimoto's disease is a common autoimmune disease characterized by chronic thyroiditis caused by anti-thyroid antibodies that can lead to hypothyroidism [1, 2]. Several studies have revealed that stroke is more common in patients with Hashimoto's disease than in the normal population [3-6]. Moreover, a recent study reported that the

\footnotetext{
* Correspondence: juntak@kuhp.kyoto-u.ac.jp

Department of Neurosurgery, National Cerebral and Cardiovascular Center, Kishibe-Shinmachi 6-1, Suita City, Osaka 564-8565, Japan
}

prevalence of anti-thyroid antibodies is higher in moyamoya disease, which is characterized by bilateral intracranial arterial stenosis (IAS), than in non-moyamoya stroke [7]. Intracranial angiographic examinations have widely been performed for Graves' disease, another autoimmune thyroid disease that can also induce ischemic stroke [8]. However, the intracranial vascular morphology and clinical course of Hashimoto's disease have poorly been documented. In the present study, we aimed to identify the

C C The Author(s). 2020 Open Access This article is licensed under a Creative Commons Attribution 4.0 International License, which permits use, sharing, adaptation, distribution and reproduction in any medium or format, as long as you give appropriate credit to the original author(s) and the source, provide a link to the Creative Commons licence, and indicate if changes were made. The images or other third party material in this article are included in the article's Creative Commons licence, unless indicated otherwise in a credit line to the material. If material is not included in the article's Creative Commons licence and your intended use is not permitted by statutory regulation or exceeds the permitted use, you will need to obtain permission directly from the copyright holder. To view a copy of this licence, visit http://creativecommons.org/licenses/by/4.0/ The Creative Commons Public Domain Dedication waiver (http://creativecommons.org/publicdomain/zero/1.0/) applies to the data made available in this article, unless otherwise stated in a credit line to the data. 
angiographic features and clinical outcomes in patients with Hashimoto's disease-associated IAS.

\section{Methods}

This retrospective study was approved by the ethics board at the authors' institute (M30-013). Individual patient consent was not required from patients. The opportunity to opt out of the study was always available to the research participants.

\section{Participants}

From January 2014 to July 2019, 390 adults (aged $\geq 20$ years) with IAS were hospitalized for neuroradiological examination. Of these, 107 patients were examined for serum autoimmune antibodies, including anti-thyroid antibodies and thyroid hormone levels. In principle, from 2014 to 2017, patients were selected for serum testing using the following criteria: (1) IAS that was unlikely to have been caused by atherosclerosis and (2) IAS that did not strictly fulfill the clinical and angiographic criteria of moyamoya disease [steno-occlusive lesion involving the terminal portion of the internal carotid arteries (ICAs) with accompanying moyamoya vessels]. We observed that the positive rate of anti-thyroid antibodies was higher than expected in IAS, regardless of their vascular morphology. Accordingly, after January 2018, the criteria were expanded to include patients whose angiographic findings were compatible with moyamoya disease. Diagnosis of Hashimoto's disease or its suspected condition was established as per the guidelines for the Diagnosis of Chronic Thyroiditis (Hashimoto's disease) established by the Japan Thyroid Association [9]. Briefly, patients were diagnosed with Hashimoto's disease when they exhibited both thyroid gland swelling without any other cause (e.g., Graves' disease) and positive antithyroid peroxidase antibodies (TPOAbs) and/or antithyroglobulin antibodies (TgAbs). Patients who exhibited positive anti-TPOAb or anti-TgAb with no thyroid goiter were suspected to have Hashimoto's disease. According to the reference level value of the institute, positive anti-TPOAb and anti-TgAb were defined as $\geq 5.61$ international unit $(\mathrm{IU}) / \mathrm{mL}$ and $\geq 4.11 \mathrm{IU} / \mathrm{mL}$, respectively.

A total of 30 patients exhibited positive anti-TPOAb or anti-TgAb; of these, 4 who exhibited hyperthyroidism and were thereafter diagnosed with Graves' disease were excluded. Thus, 26 patients (24.3\%) were finally included in this study (definite 1, suspected 26).

\section{Angiographic classification of IAS (Fig. 1)}

All patients underwent digital subtraction (DS) angiography. The affected hemispheres were classified into five groups according to the location of the arterial stenoocclusion and development of abnormal collateral vessels (moyamoya vessels):
Group A: stenosis/occlusion of the ICA bifurcation with moyamoya vessels

Group B: stenosis/occlusion of the M1/A1 with moyamoya vessels

Group C: stenosis/occlusion of the ICA bifurcation without moyamoya vessels

Group D: stenosis/occlusion of the M1/A1 without moyamoya vessels

Group E: stenosis/occlusion of the M2-M4/A2-A4 without moyamoya vessels

All the affected hemispheres exhibited IAS in the anterior circulations. No hemisphere exhibited proximal ICA stenosis with normal ICA bifurcation. No hemisphere exhibited both stenosis/occlusion of the M2-M4/ A2-A4 and moyamoya vessels.

The hemispheres in Groups A and B were defined as the moyamoya vessels positive (MM) type, whereas those in Groups $\mathrm{C}-\mathrm{E}$ were defined as the moyamoya vessels negative (non-MM) type. In the MM type hemispheres, the development of collateral vessels (periventricular anastomosis) was evaluated according to the criteria of the supplemental studies of the Japan Adult Moyamoya Trial (Table 1) [10, 11].

\section{Clinical management and follow-up}

All the hemispheres were evaluated using single photon emission computed tomography (SPECT) and/or positron emission computed tomography (PET) at the time of diagnosis. Direct bypass surgery [superficial temporal artery (STA)-middle cerebral artery (MCA) anastomosis] was performed within 3 months after diagnosis if the patient exhibited severe hemodynamic failure. All patients were semiannually followed-up using serial magnetic resonance (MR) imaging and monitored for stenosis progression and cerebrovascular events, including transient ischemic attacks (TIAs), intracranial hemorrhage, and cerebral infarction. If MR angiography detected stenosis progression, DS angiography was performed to confirm this finding. The time from diagnosis to these incidents was compared between the MM type and non-MM type hemispheres. This duration was also compared between patients with hypothyroidism (T4 $\leq 1.1 \mathrm{ng} / \mathrm{dL}$ and/or TSH $\geq 5.5 \mu \mathrm{U} /$ $\mathrm{mL}$ ) and those without hypothyroidism. The modified Rankin scale (mRS) scores of the patients were evaluated at the final follow-up.

\section{Statistical analyses}

A t-test, Mann-Whitney's U test, chi-squared test, or Fisher's exact test was used for comparing the baseline characteristics of the two groups. Kaplan-Meier survival analysis with a log-rank test was performed for comparing the length of time without an adverse event (IAS progression, ischemic events, and intracranial hemorrhage) in 


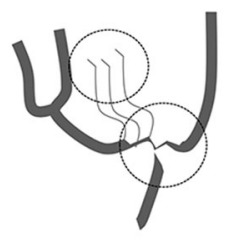

Group A: 4 (9.5\%)

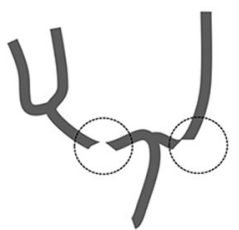

Group D: 16 (38.1\%)

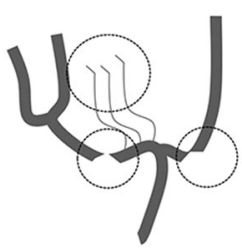

Group B: 13 (31\%)

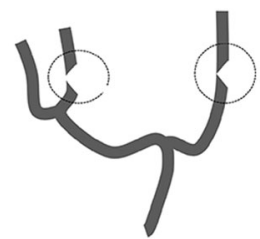

Group E: 2 (4.8\%)

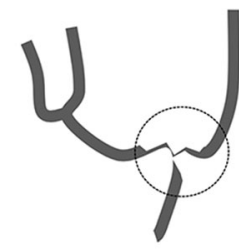

Group C: 7 (16.7\%)

Fig. 1 Angiographic classification of the 42 affected hemispheres. Group A: stenosis/occlusion of IC bifurcation with moyamoya vessels. Group B: stenosis/occlusion of M1/A1 with moyamoya vessels. Group C: stenosis/occlusion of IC bifurcation without moyamoya vessels. Group D: stenosis/ occlusion of M1/A1 without moyamoya vessels. Group E: stenosis/occlusion of M2-4/A2-4 without moyamoya vessels

each group. Two-sided $P$ values $<0.05$ were considered to indicate statistical significance. All analyses were performed using JMP software, ver. 11 (SAS Institution, Cary, USA).

\section{Results}

\section{Patient characteristics}

Table 2 shows the baseline characteristics of the 26 patients. The mean age \pm standard deviation (SD) was $56.4 \pm 13.2$ years (range $35-79$ years). The initial presentation was TIAs in 12 patients, cerebral infarction in 7 , and intracranial hemorrhage in $3 ; 4$ patients were asymptomatic.

\section{Angiographic features and clinical presentations at diagnosis}

DS angiography at diagnosis demonstrated bilateral IAS in $16(61.5 \%)$ patients and unilateral IAS in 10 (38.5\%) patients, yielding 42 affected hemispheres. Based on the site of stenosis and development of moyamoya vessels, these hemispheres were classified as follows: Group A, 4; Group B, 13; Group C, 7; Group D, 16; and Group E, 2 (Figs. 1 and 2). Further, 17 hemispheres of Groups A and $\mathrm{B}$ were defined as the MM type and 25 hemispheres of Groups $\mathrm{C}-\mathrm{E}$ were defined as the non-MM type. In 3 patients, the MM type and non-MM type hemispheres coexisted.

Table 3 lists the characteristics of the 42 affected hemispheres. No differences were observed in terms of age and sex between the MM type and non-MM type hemispheres. Hypothyroidism was identified in 11 and 14 of the MM type and non-MM type hemispheres, respectively $(p=0.75)$. Ischemic presentation at onset was significantly more common in non-MM type hemispheres than in the MM type hemispheres $(92.0 \%$ vs. $35.3 \%, p<0.0001)$, whereas the hemorrhagic presentation was significantly more common in the MM type hemispheres ( $0 \%$ vs. $35.3 \%, p=0.0013)$.

\section{Initial surgical intervention at the diagnosis of IAS}

Based on the ischemic symptoms and severe hemodynamic failure, 6 (35.3\%) MM type hemispheres and 3 (12.0\%) non-MM type hemispheres underwent STAMCA anastomosis within 3 months after diagnosis, whereas conservative management was initiated for the remaining hemispheres. Antiplatelet drugs were prescribed for 17 (65.4\%) patients.

Table 1 Definition of positive development of periventricular anastomosis [10, 11]

\begin{tabular}{|c|c|}
\hline Lenticulostriate anastomosis & - Extreme dilation and extension of the lenticulostriate arteries beyond the level of the pericallosal artery \\
\hline Thalamic anastomosis & $\begin{array}{l}\text { - Extreme dilation and extension of the thalamic perforators beyond the position of the medial posterior } \\
\text { choroidal artery }\end{array}$ \\
\hline \multirow[t]{3}{*}{ Choroidal anastomosis } & $\begin{array}{l}\text { - Extreme dilation and extension of the choroidal artery with sudden deviation from the shape of the lateral } \\
\text { ventricle at its peripheral portion to connect to the medullary artery in the anteroposterior view. }\end{array}$ \\
\hline & $\begin{array}{l}\text { - Extreme extension of the anterior choroidal or lateral posterior choroidal artery beyond the atrium of the } \\
\text { lateral ventricle to reach the body of the lateral ventricle. }\end{array}$ \\
\hline & - Connection of the medial posterior choroidal artery to the pericallosal artery by penetrating the corpus callosur \\
\hline
\end{tabular}


Table 2 Characteristics of 26 IAS patients with positive antithyroid antibodies

\begin{tabular}{ll}
\hline & No. of patients \\
\hline Number of patients & 26 \\
Mean age at diagnosis \pm SD (range) & $56.4 \pm 13.2(35-79)$ \\
Females & $19(73.1 \%)$ \\
Bilateral & $16(61.5 \%)$ \\
Symptoms at onset & \\
TIA & $12(46.2 \%)$ \\
Cerebral infarction & $7(26.9 \%)$ \\
Hemorrhage & $3(11.5 \%)$ \\
$\quad$ Asymptomatic & $4(15.4 \%)$ \\
Hypertension & $19(73.1 \%)$ \\
Diabetes mellitus & $5(19.2 \%)$ \\
Hyperlipidemia & $10(38.5 \%)$ \\
Hypothyroidism & $16(61.5 \%)$ \\
Family history & \\
Moyamoya disease & $3(11.5 \%)$ \\
Thyroid disease & $1(3.8 \%)$ \\
\hline
\end{tabular}

IAS progression and cerebrovascular events during the follow-up periods

Figure 3 shows the Kaplan-Meier curve of the MM type and non-MM type hemispheres with regard to IAS progression. During the follow-up periods of $2.5 \pm 2.9$ years (mean $\pm \mathrm{SD}$ ), no IAS progression was confirmed in the MM type hemispheres. However, 8 of the non-MM type hemispheres showed IAS progression at $2.9 \pm 3.8$ years after diagnosis (10.5\%/year); this was significantly more common than that in the MM type hemispheres $(p=$ 0.041). A transition from non-MM to MM or that from MM to non-MM type was not observed during the follow-up period. Multivariate analysis with calculation of hazard ratio could not be performed because the event rate was zero in the MM type hemispheres.

Figure 4 shows the Kaplan-Meier curve for ischemic (A) and hemorrhagic (B) events during the follow-up periods. Of the non-MM type hemispheres, 5 exhibited ischemic attacks (6.6\%/year), whereas no ischemic event was observed in the MM type hemispheres $(p=0.080)$. Owing to ischemic events or severe hemodynamic deterioration, STA-MCA anastomosis was newly undertaken for 8 non-MM type hemispheres at $1.4 \pm 0.64 \mathrm{y}$ after the diagnosis. There were no additional bypass surgeries in the MM type hemispheres except for 1 case with an "initially planned" bypass that was postponed until 7 months after diagnosis because of postsurgical

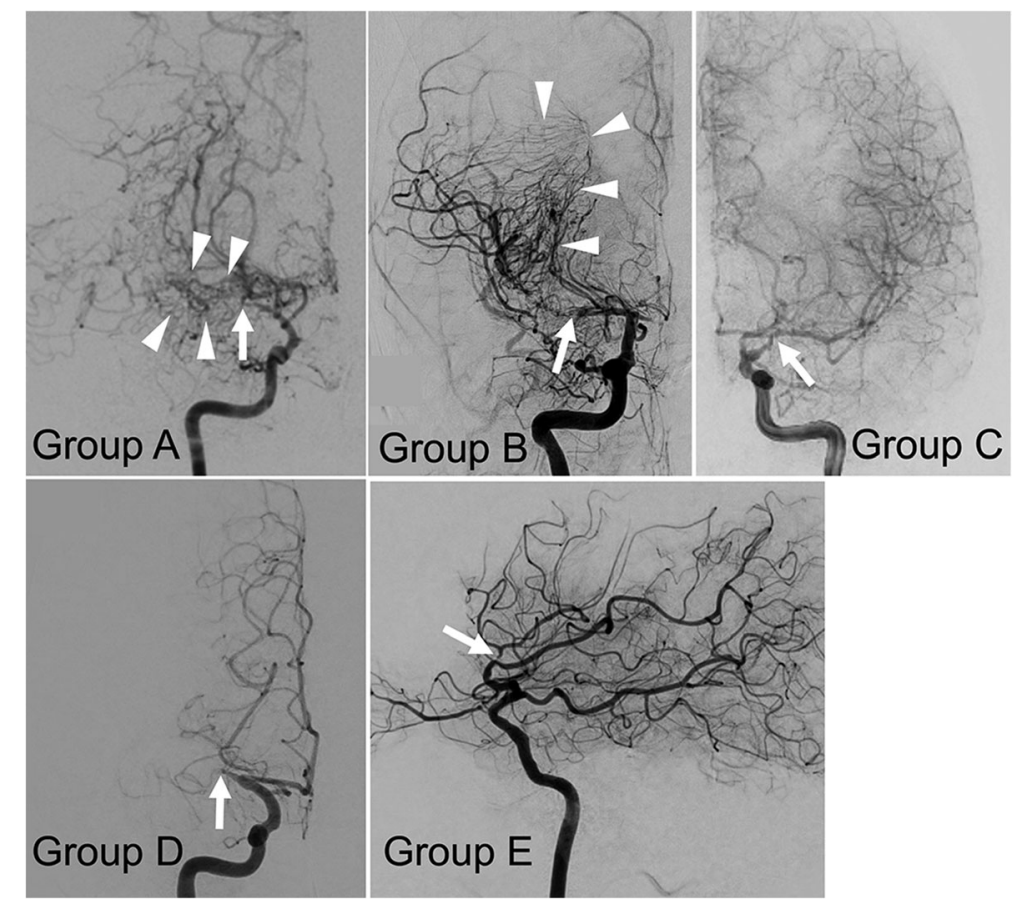

Fig. 2 Representative DS angiograms of Groups A-E. Arrows: location of the arterial stenosis. Arrowheads: abnormal collateral vessels (moyamoya vessels) 
Table 3 Characteristics of 42 affected hemispheres

\begin{tabular}{|c|c|c|c|}
\hline & $\begin{array}{l}\text { MM type } \\
\text { (Group A, B) }\end{array}$ & $\begin{array}{l}\text { Non-MM type } \\
\text { (Group } C, D \text {, and } E \text { ) }\end{array}$ & $P$ value \\
\hline Number of hemispheres & $17(40.5 \%)$ & $25(59.5 \%)$ & \\
\hline Mean age at diagnosis \pm SD & $58.4 \pm 15.3$ & $55.0 \pm 11.8$ & 0.43 \\
\hline Females & $14(82.4 \%)$ & $18(72.0 \%)$ & 0.49 \\
\hline Coexisting contralateral IAS & $13(76.5 \%)$ & $19(76.0 \%)$ & 0.97 \\
\hline \multicolumn{4}{|l|}{ Symptoms at onset } \\
\hline Ischemic & $6(35.3 \%)$ & $23(92.0 \%)$ & $<0.0001$ \\
\hline Hemorrhage & $6(35.3 \%)$ & $0(0 \%)$ & 0.0013 \\
\hline Asymptomatic & $5(29.4 \%)$ & $2(8.0) \%$ & 0.068 \\
\hline Hypothyroidism & $11(64.7 \%)$ & $14(56.0 \%)$ & 0.75 \\
\hline
\end{tabular}

complications in the opposite hemisphere. Overall, surgical intervention was provided for 7 (41.2\%) of the MM type hemispheres and 11 (44\%) of the non-MM type hemispheres during the entire period ( $p=0.86$ ). Four of the MM type hemispheres had an intracerebral hemorrhage (12.0\%/ year), whereas none of the non-MM type hemispheres developed a hemorrhage $(p=0.012)$. Multivariate analyses for ischemic and hemorrhagic events were not conducted because the event rate was zero in the MM type for the former and in the non-MM type for the latter. Kaplan-Meier analysis showed no differences between the hemispheres with hypothyroidism and those without hypothyroidism in terms of IAS progression $(p=$ $0.078)$, ischemic events $(p=0.83)$, and intracranial hemorrhaging $(p=0.39)$.

The mRS in the 26 patients at the time of final followup was 0 in 17, 1 in 5, 2 in 1, and 4 in 3 patients. Poor outcome (indicated by an mRS score of 4) was attributed to intracranial hemorrhaging in 2 of the MM type hemispheres and ischemic stroke in 1 of the non-MM type hemispheres during the follow-up period.

\section{Development of periventricular anastomosis in the MM type hemispheres}

Figure 5 demonstrates the positive rate of each periventricular anastomosis in the $17 \mathrm{MM}$ type hemispheres as per the criteria proposed in the supplement studies of the Japan Adult Moyamoya Trial $[10,11]$. The rate of positive choroidal anastomosis, which has recently been described as a factor associated with posterior hemorrhage at a high risk of re-bleeding and a predictor of recurrent and de novo intracranial hemorrhage in moyamoya disease [10-12], was extremely high $(76.5 \%)$ compared with those of lenticulostriate and thalamic anastomosis.

\section{IAS progression}

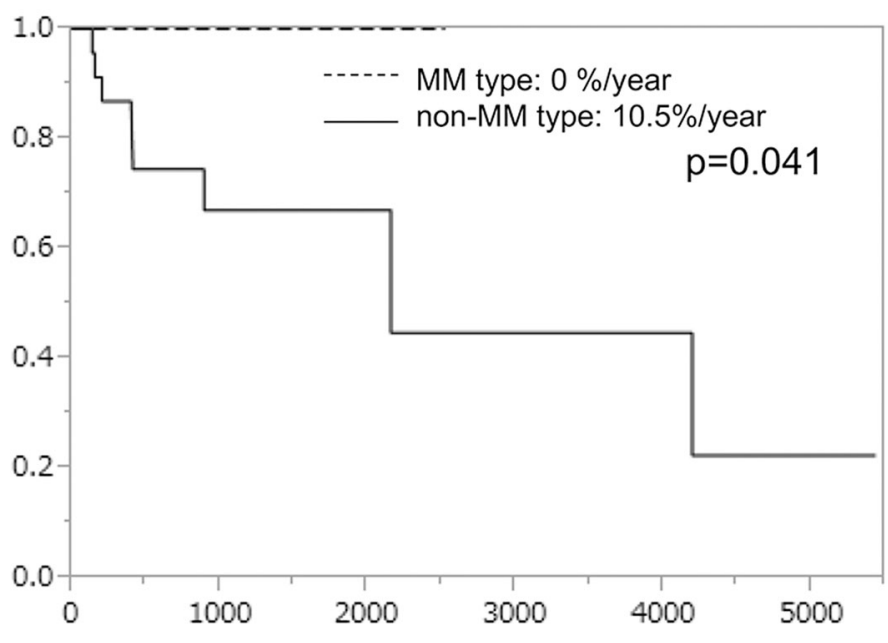

Fig. 3 Kaplan-Meier curves for IAS progression in MM type and non-MM type hemispheres 

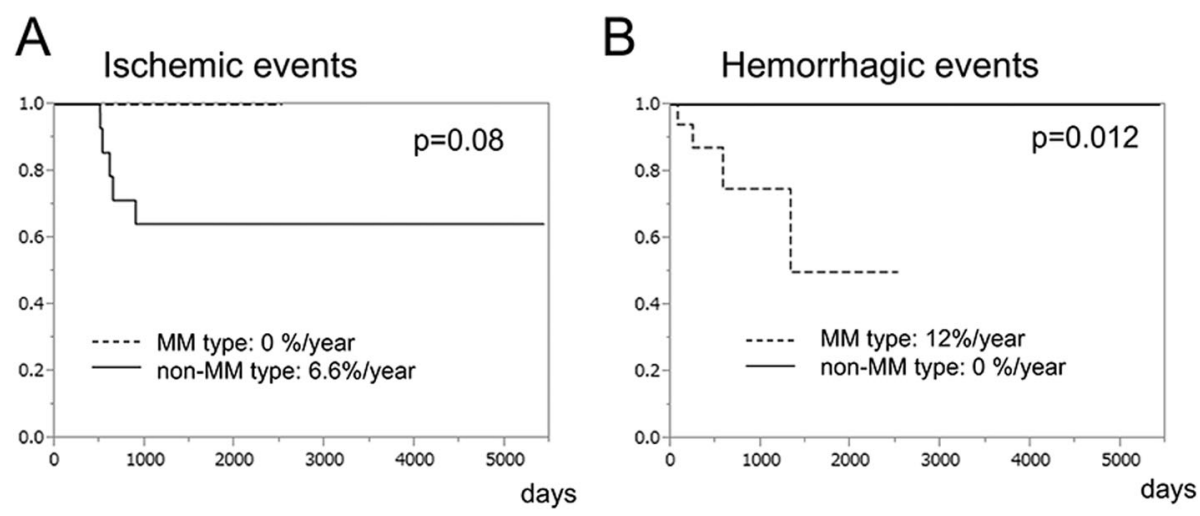

Fig. 4 Kaplan-Meier curves for cerebrovascular events in the MM type and non-MM type hemispheres. (a) Ischemic and (b) hemorrhagic events

\section{Discussion}

Hashimoto's disease (chronic thyroiditis) is a common autoimmune disease that is characterized by a reduction in thyroid hormone level, thyroid goiter, and symptoms, including systemic edema, dyslipidemia, and increased body weight [1]. Chronic inflammation is attributed to the autoimmune process caused by anti-TPOAb and/or anti-TgAb $[1,2]$. The prevalence is considerably high and is reportedly 46 cases/1000 [4]. In the Japanese population, anti-thyroid antibodies are reportedly present in $10.2-14.2 \%$ of women and $6-7.2 \%$ of men [13]. Several studies have recently revealed that stroke is more common in patients with Hashimoto's disease than in the general population [3-6]. However, their intracranial vascular morphology and clinical courses have poorly been documented.

To the best of the authors' knowledge, this is the first study to analyze detailed angiographic features and clinical courses in Hashimoto's disease-associated IAS. A stenotic lesion was visible at the IC bifurcation, which is commonly affected in true moyamoya disease, whereas the stenosis was also observed in M1, A1, or distal ACA/ MCA with an intact IC bifurcation. The affected hemispheres could be dichotomized into the MM type and non-MM type with respect to abnormal collateral vessels.

Several autoimmune disorders can reportedly induce IAS. These include Graves' disease, systematic lupus erythematosus, and Sjögren syndrome [8, 14-17]. In Graves' disease, which results in hyperthyroidism, recent reports have highlighted the angiographic features of IAS, demonstrating that the involvement of the IC bifurcation and moyamoya vessels can be confirmed in 79.7 and $75.9 \%$ of the cases, respectively [8]. However, in other autoimmune diseases, including Hashimoto's disease, detailed cerebral angiographic features have not been described in the literature to date.

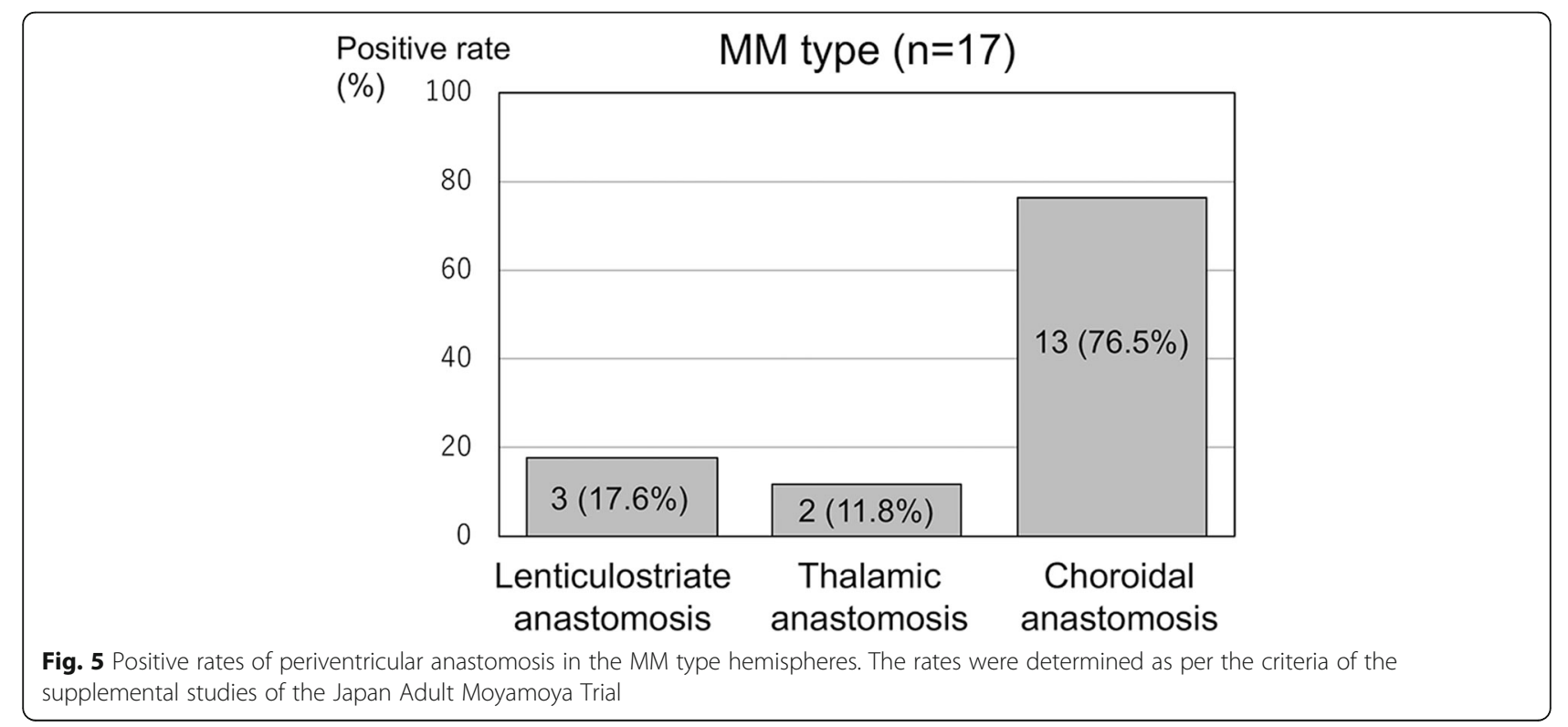


In the present study, the angiographic features of the non-MM type hemispheres were different from those of moyamoya disease, suggesting that this type is Hashimoto's disease-associated vasculopathy. However, it is unclear whether the MM type is an independent entity associated with Hashimoto's disease or it coincidentally coexists with true moyamoya disease and Hashimoto's disease. This question arises because the prevalence of Hashimoto's disease is considerably high in the population $[1,18,19]$. This should be considered particularly for Group A, consistent with the diagnostic criteria of moyamoya disease. However, Group B that accounted for $76 \%$ of the MM type hemispheres, lacked stenosis of the IC bifurcation, indicating that Hashimoto's diseaseassociated MM type can exist as an independent entity.

It is noteworthy that the non-MM type hemispheres were at a significantly higher risk of stenosis progression than the MM type hemispheres. Furthermore, ischemic events were observed only in the non-MM type hemispheres during the follow-up periods. In the present study, bypass surgery was performed for 4 non-MM type hemispheres during the follow-up period because of asymptomatic stenosis progression. This suggested that the ischemic risk would have been higher if surgery was not performed. In the Kaplan-Meier curve analysis for ischemic events, the additional bypass surgeries were not considered (Fig. 4a). The result was comparable when surgical intervention was considered as "censoring" ( $p=$ 0.084).

Further, it is noteworthy that MM type hemispheres had a high risk of intracranial hemorrhage. Hemorrhage at the initial presentation was observed only in MM type hemispheres. Furthermore, only MM type hemispheres developed hemorrhaging during the follow-up periods with an annual rate of $12.0 \%$ /year. The reported annual re-bleeding rate of hemorrhagic moyamoya disease is 7.1$7.6 \%$, and recent studies have highlighted the importance of periventricular anastomosis, particularly on choroidal anastomosis as a risk factor of recurrent and de novo intracranial hemorrhage in moyamoya disease [10-12, 20-22]. In the present study, $76.5 \%$ of the MM type hemispheres exhibited positive choroidal anastomosis. This may explain the high hemorrhagic rate in MM type hemispheres in Hashimoto's disease-associated IAS.

The mechanism of IAS in Hashimoto's disease has been poorly documented by previous studies. In Graves' disease, a T-cell-mediated immunological mechanism and/or sympathetic-nerve-mediated vasculopathy are presumed to cause IAS [3, 23, 24]. An elevated cerebral metabolism of oxygen and hypercoagulability caused by elevated thyroid hormones (hyperthyroidism) has the ability to aggravate cerebral ischemia [3, 23, 24]. However, for Hashimoto's disease, no promising hypothesis explaining the progression of vascular stenosis has emerged. Recent reports have documented a high rate of positive anti-thyroid antibodies in moyamoya disease [5, 23]. Kim SJ et al. compared the positive rate of antimicrosomal antibodies (AMA) that is equivalent to TPOAb among the following three groups: moyamoya disease, non-moyamoya stroke, and normal controls [5]. The prevalence of AMA was significantly higher in the presence of moyamoya disease. Moreover, Tashiro et al. have examined the association between human leukocyte antigen (HLA) and moyamoya disease [7]. They showed that HLA-DRB1*04:10 was a risk allele for moyamoya disease and that thyroid diseases, such as Graves' and Hashimoto's disease, were more common in HLADRB1*04:10-positive patients with moyamoya disease than in HLA-DRB1*04:10-negative patients. These data suggested a potential immunological mechanism in Hashimoto's disease-associated IAS. Hypothyroidism itself reportedly increases the risk for cardiovascular events and atherosclerosis [25, 26]. However, in the present study, decreased thyroid hormone levels were not associated with cerebrovascular events or IAS progression. Further studies are required to elucidate the pathophysiology of Hashimoto's disease-associated IAS.

There are several limitations of the present study. First, this was a retrospective cohort study with a relatively small sample size, and selection bias could not be eliminated because screening for anti-thyroid antibodies was not performed for all patients with IAS. Second, the follow-up periods were insufficient. Third, analyses of the subsequent vascular events were not based on a purely natural course because some patients underwent bypass surgery at the beginning of the follow-up period. Despite the limitations, this study showed the outcomes under a certain management strategy, including surgical intervention for the hemodynamically impaired patients.

\section{Conclusions}

Hashimoto's disease-associated IAS exhibits several types of angiographic morphologies. Non-MM type hemispheres are at risk of IAS progression and ischemic events during the short term, whereas MM type hemispheres can develop subsequent intracranial hemorrhaging at a high rate. Although the pathophysiology of Hashimoto's disease-associated IAS requires further elucidation, these results strongly suggest the importance of screening for anti-thyroid antibodies and careful management based on vascular morphology in adult patients with IAS.

\section{Acknowledgements \\ Not applicable.}

Authors' contributions

EH and JCT designed this study, analyzed/interpreted the all patient data, and wrote the manuscript. MN, HM, and TS contributed to this study in data acquisition. The authors read and approved the final manuscript. 


\section{Funding}

Not applicable.

\section{Availability of data and materials}

The datasets generated and/or analyzed during the current study are not publicly available due to personal information included, but are available from the corresponding author on reasonable request.

\section{Ethics approval and consent to participate}

All procedures performed in studies involving human participants were in accordance with the ethical standards of the research committee of National Cerebral and Cardiovascular Center, and with the 1964 Helsinki Declaration and its later amendments or comparable ethical standards. Because no information that would allow identification of the patient was collected, individual patient consent was not required from patients. The opportunity to opt out of the study was always available to the research participants. This retrospective study was approved by the Ethics Committee of the National Cerebral and Cardiovascular Center (IRB approval no. M30-013).

\section{Consent for publication}

Not applicable.

\section{Competing interests}

The authors declare that they have no competing interests.

Received: 16 June 2020 Accepted: 8 September 2020

\section{Published online: 14 September 2020}

\section{References}

1. Caturegli P, De Remigis A, Rose NR. Hashimoto thyroiditis: clinical and diagnostic criteria. Autoimmun Rev. 2014;13:391-7.

2. Pyzik A, Grywalska E, Matyjaszek-Matuszek B, Roliński J. Immune disorders in Hashimoto's thyroiditis: what do we know so far? J Immunol Res. 2015;2015: 979167

3. Chen JB, Lei D, He M, Sun H, Liu YZH. Clinical features and disease progression in moyamoya disease patients with graves disease. J Neurosurg. 2015;123:1608

4. Karch A, Thomas SL. Autoimmune thyroiditis as a risk factor for stroke: a historical cohort study. Neurology. 2014;82:1643-52.

5. Kim SJ, Heo KG, Shin HY, Bang OY, Kim G-M, Chung C-S. Association of Thyroid Autoantibodies with Moyamoya-Type Cerebrovascular Disease a Prospective Study. Stroke. 2010:41:173-6.

6. Zöller B, Li X, Sundquist J, Sundquist K. Risk of subsequent coronary heart disease in patients hospitalized for immune-mediated diseases: a nationwide follow-up study from Sweden. PLoS One. 2012;7(3):1-9.

7. Tashiro R, Niizuma K, Khor SS, Tokunaga K, Fujimura M, Sakata H, et al. Identification of HLA-DRB1*04:10 allele as risk allele for Japanese moyamoya disease and its association with autoimmune thyroid disease: a case-control study. PLoS One. 2019;14:1-12.

8. Shah NH, Khandelwal P, Gordon-Perue G, Shah AH, Barbarite E, Ortiz G, et al. Acute thyrotoxicosis of graves disease associated with Moyamoya vasculopathy and stroke in Latin American women: a case series and review of the literature. World Neurosurg. 2016;92:95-107.

9. Japan thyroid association. Guidelines for the diagnosis of chronic thyroiditis. 2010 http://www.japanthyroid.jp/doctor/guideline/englis. Accessed 22 May 2020

10. Funaki T, Takahashi JC, Houkin K, Kuroda S, Takeuchi S, Fujimura M, et al. Angiographic features of hemorrhagic moyamoya disease with high recurrence risk: a supplementary analysis of the Japan adult Moyamoya trial. J Neurosurg. 2018;128:777-84.

11. Funaki T, Takahashi JC, Houkin K, Kuroda S, Takeuchi S, Fujimura M, et al. High rebleeding risk associated with choroidal collateral vessels in hemorrhagic moyamoya disease: analysis of a nonsurgical cohort in the Japan adult Moyamoya trial. J Neurosurg. 2019;130:525-30.

12. Funaki T, Takahashi JC, Houkin K, Kuroda S, Fujimura M, Tomata Y, et al. Effect of choroidal collateral vessels on de novo hemorrhage in moyamoya disease: analysis of nonhemorrhagic hemispheres in the Japan adult Moyamoya trial. J Neurosurg. 2020;132:408-14.

13. McLeod DSA, Cooper DS. The incidence and prevalence of thyroid autoimmunity. Endocrine. 2012;42:252-65.
14. Lee MC, Lee YS, Ryu HS, Lee WS, Park WK, Chang TW. Systemic lupus erythematosus associated with familial moyamoya disease. Korean J Intern Med. 2003;18:244-7.

15. Matsuki Y, Kawakami M, Ishizuka T, Kawatuchi Y, Hidaka T, Suzuki K, Nakamura H. SLE and Sjogren s syndrome associated with unilateral Moyamoya vessels in cerebral arteries. Scand J Rheumatol. 1997;26(5):392-4.

16. Nagahiro S, Mantani A, Yamada K, Ushio Y. Multiple cerebral arterial occlusions in a young patient with Sjögren's syndrome: case report. Neurosurgery. 1996;38:592-5.

17. Sakata H, Fujimura M, Sato K, Shimizu H, Tominaga T. Efficacy of extracranial-intracranial bypass for progressive middle cerebral artery occlusion associated with active Sjögren's syndrome: case report. J Stroke Cerebrovasc Dis. 2014:23:e399-402

18. Hollowell JG, Staehling NW, Dana Flanders W, Harry Hannon W, Gunter EW, Spencer CA, et al. Serum TSH, T4, and thyroid antibodies in the United States population (1988 to 1994): National Health and nutrition examination survey (NHANES III). J Clin Endocrinol Metab. 2002;87:489-99.

19. Jacobson DL, Gange SJ, Rose NRGN. Epidemiology and estimated population burden of selected autoimmune diseases in the United States. Clin Immunol Immunopathol. 1997:84(3):223-43.

20. Funaki T, Fushimi Y, Takahashi JC, Takagi Y, Araki Y, Yoshida K, et al. Visualization of periventricular collaterals in moyamoya disease with flowsensitive black-blood magnetic resonance angiography: Preliminary experience. Neurol Med Chir (Tokyo). 2015:55:204-9.

21. Funaki T, Takahashi JC, Yoshida K, Takagi Y, Fushimi Y, Kikuchi T, et al. Periventricular anastomosis in moyamoya disease: detecting fragile collateral vessels with MR angiography. J Neurosurg. 2016;124:1766-72.

22. Morioka M, Hamada Jl, Kawano T, Todaka T, Yano S, Kai Y, et al. Angiographic dilatation and branch extension of the anterior choroidal and posterior communicating arteries are predictors of hemorrhage in adult moyamoya patients. Stroke. 2003;34:90-5.

23. Li H, Zhang Z-S, Dong Z-N, Ma M-J, Yang W-Z, Han C, et al. Increased thyroid function and elevated thyroid autoantibodies in pediatric patients with Moyamoya disease a case-control study. Stroke. 2011;42:1138-9.

24. Utku U, Asil T, Celik Y, Tucer D. Reversible MR angiographic findings in a patient with autoimmune graves disease. Am J Neuroradiol. 2004;25:1541-3.

25. Hak AE, Pols HA, Visser TJ, Drexhage HA, Hofman A, Witteman JC Subclinical hypothyroidism is an independent risk factor for atherosclerosis and myocardial infarction in elderly women: the Rotterdam study. Ann Intern Med. 2000;132:270-8.

26. Ochs N, Auer R, Bauer DC, Nanchen D, Gussekloo J, Cornuz J, et al. Metaanalysis: subclinical thyroid dysfunction and the risk for coronary heart disease and mortality. Ann Intern Med. 2008;148:832-45.

\section{Publisher's Note}

Springer Nature remains neutral with regard to jurisdictional claims in published maps and institutional affiliations.

Ready to submit your research? Choose BMC and benefit from:

- fast, convenient online submission

- thorough peer review by experienced researchers in your field

- rapid publication on acceptance

- support for research data, including large and complex data types

- gold Open Access which fosters wider collaboration and increased citations

- maximum visibility for your research: over $100 \mathrm{M}$ website views per year

At $\mathrm{BMC}$, research is always in progress.

Learn more biomedcentral.com/submissions 